듬 SCHOOL of GRADUATE STUDIES

EAST TENNESSEE STATE UNIVERSITY
East Tennessee State University Digital Commons@ East Tennessee State University

$8-2018$

\title{
Cluster Set Loading in The Back Squat: Kinetic and Kinematic Implications
}

Alexander Wetmore

East Tennessee State University

Follow this and additional works at: https://dc.etsu.edu/etd

Part of the Sports Sciences Commons

\section{Recommended Citation}

Wetmore, Alexander, "Cluster Set Loading in The Back Squat: Kinetic and Kinematic Implications" (2018). Electronic Theses and Dissertations. Paper 3437. https://dc.etsu.edu/etd/3437

This Thesis - Open Access is brought to you for free and open access by the Student Works at Digital Commons @ East Tennessee State University. It has been accepted for inclusion in Electronic Theses and Dissertations by an authorized administrator of Digital Commons@ East Tennessee State

University. For more information, please contact digilib@etsu.edu. 
Cluster Set Loading in The Back Squat: Kinetic and Kinematic Implications

\author{
A thesis \\ presented to \\ the faculty of the Department of Sport, Exercise, Recreation and Kinesiology \\ East Tennessee State University
}

In partial fulfillment

of the requirements for the degree

Master of Science in Sport Science and Coach Education

by

Alexander B. Wetmore

August 2018

Michael H. Stone, Ph.D., Chair

Brad H. DeWeese, Ph.D., Committee Member

Kimitake Sato, Ph.D., Committee Member

Keywords: Training, Rest, Strength and Conditioning, Performance 


\section{ABSTRACT \\ Cluster Set Loading in The Back Squat: Kinetic and Kinematic Implications}

by

\section{Alexander B. Wetmore}

The purpose of this study was to investigate the kinetic and kinematic implications of cluster loading as a resistance training programming tactic. Cluster loading involves introducing rest during a set which may allow athletes to train at higher absolute intensities. Eleven trained males were recruited for this study. Subjects completed two testing sessions consisting of three sets of five back squats at $80 \%$ of their one repetition maximum. Cluster loading included 30s of inter-repetition rest. All testing was done on dualforce plates with four linear position transducers. Paired sample t-tests were used to determine differences between conditions with Cohen's d effect sizes describing the magnitude of change between conditions. Both conditions had similar values for peak force and average force. Cluster loading had significantly higher power and velocity outputs, shorter times to peak power and velocity as well as greater maintenance of time to peak power. These results suggest cluster loading may be superior to traditional loading when maintaining power output and timepoint variables is the desired outcome of training. 
Copyright 2018 by Alexander B. Wetmore

All Rights Reserved 


\section{DEDICATION}

This thesis is dedicated to my family who has supported me through everything and given me the opportunity to chase my dreams. To my fiancée, Courtney, thank you for the countless times you have picked me up when I'm feeling overwhelmed. Words are inadequate to describe how much your love and support means to me. I love you. 


\section{ACKNOWLEDGMENTS}

I would like to express my gratitude to the following people:

Dr. Michael H. Stone, thank you for your guidance through this process and helping mentor me as a researcher. Your commitment to excellence is truly inspiring.

Dr. Brad H. DeWeese, thank you for challenging me to grow as a coach, scientist, and person. The critical thinking skills you have helped me develop will prove essential for many years to come.

Dr. Kimi Sato, thank you for always asking the necessary questions and helping me determine why research is meaningful and pushing me to make an impact with my work.

Dr. Matt Sams, thank you for giving me my first experience in sport science and for your guidance throughout my time at ETSU.

John Wagle, thank you for taking me under your wing, always providing prospective, and your countless hours of editing that went into this process.

Coach Bo Oshoniyi, David Lilly, and Brad Camp, thank you for trusting me to work with your athletes and showing me what it takes to be a great coach. 
ABSTRACT

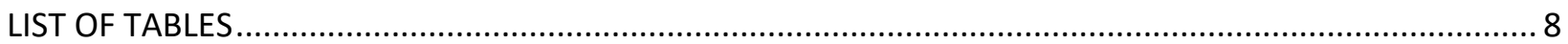

LIST OF FIGURES

Chapter

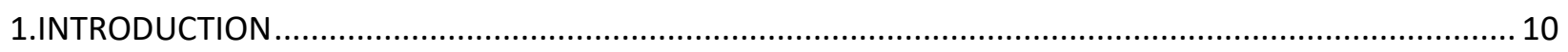

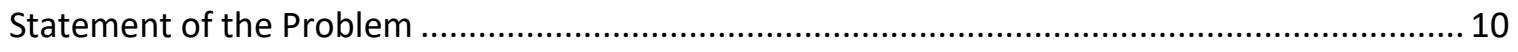

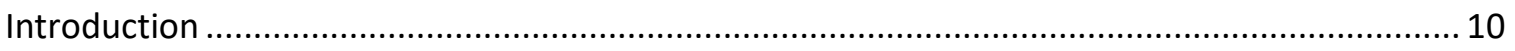

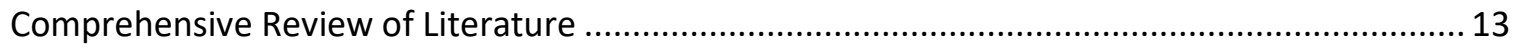

2. CLUSTER SET LOADING IN THE BACK SQUAT: KINETIC AND KINEMATIC IMPLICATIONS ...................... 19

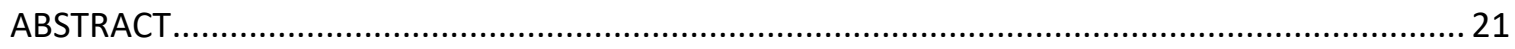

INTRODUCTION

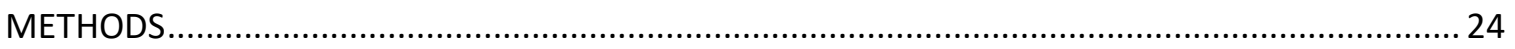

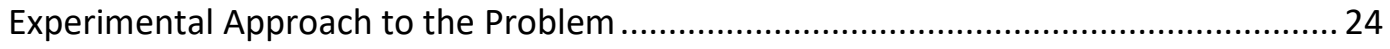

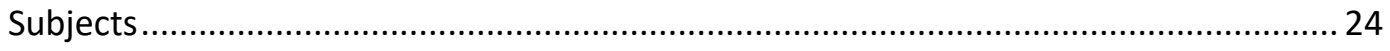

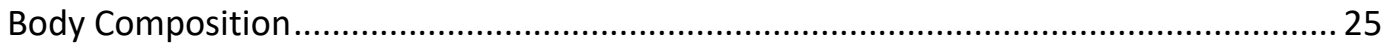

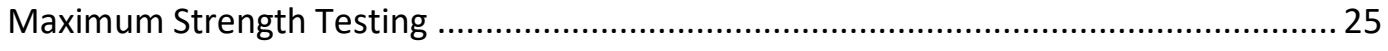

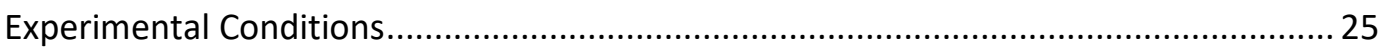




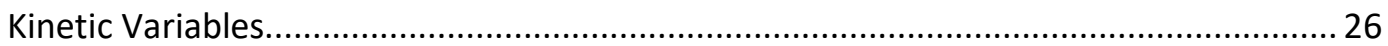

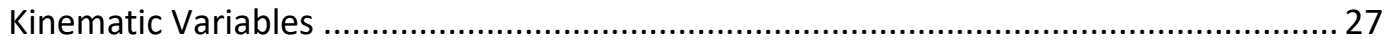

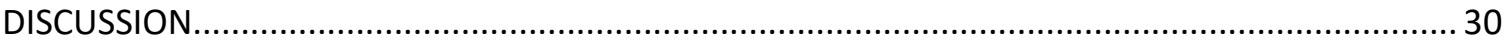

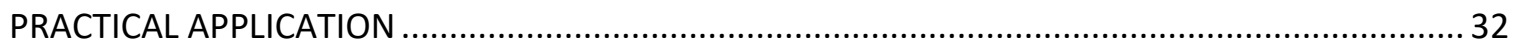

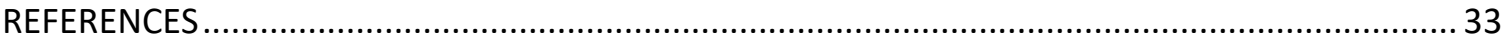

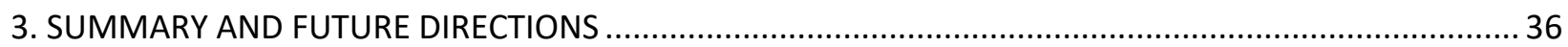

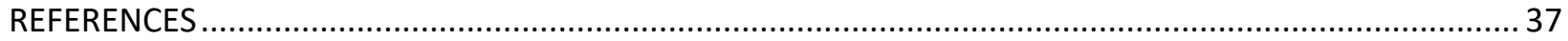

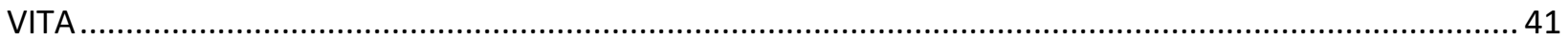




\section{LIST OF TABLES}

Table

Page

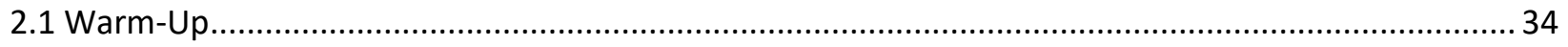

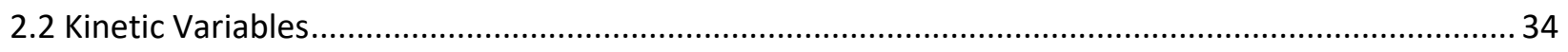

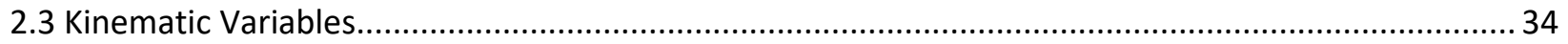




\section{LIST OF FIGURES}

Figure

Page

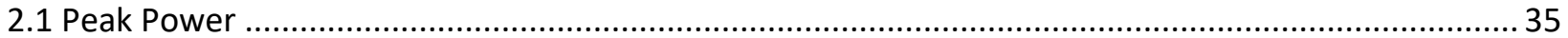

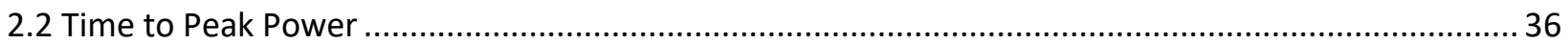

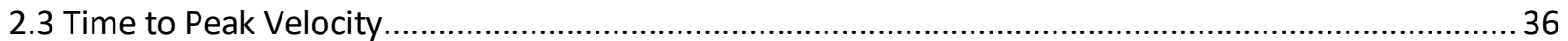


CHAPTER 1

\section{INTRODUCTION}

\section{Statement of the Problem}

Cluster loading has been previously investigated but there is a need for a full description of the kinetic and kinematic effects of this programming tactic. The purpose of this study was to investigate the effects of cluster loading on both kinetic and kinematic outputs in order to better understand its application as a resistance training programming tactic.

\section{$\underline{\text { Introduction }}$}

Over the past several years, new advanced training (AT) methods have been proposed. These AT include accentuated eccentric loading, contrast sets, complex sets and cluster sets. Cluster sets (CS) use short rest periods between repetitions as well as typical rest periods between sets (Haff et al., 2003; Haff et al., 2016). According to the SAID (specific adaptation to imposed demands) principle, changing variables within the application of an exercise elicits a specific response and subsequent adaptation given adequate recovery is provided (Stone et al., 2007). Thus, intra-set rest theoretically could allow CS to induce different adaptations to training by allowing for heavier loading at the same training volume (strength), potentiate explosiveness and power adaptations by maintenance of forces, rate of development (RFD), velocity, or power at a given load (Denton \& Cronin, 2006; Haff et al., 2003; Hansen et al., 2011a; Hansen et al., 2011b; Hardee et al., 2012b; Iglesias-Soler et al., 2016; Joy et al., 2013; Lawton et al., 2006; Moir et al., 2013; Moreno et al., 2014; Oliver et al., 2016; Oliver et al., 2015; Tuffano et al., 2016a; Tuffano et al., 2016b). Thus, CS training could be useful for a variety of purposes such as enhancing the training effect by offering a greater stimulus or varying the stimulus to promote further adaptation. For example, training over a few years with little variation, such as can occur with 
maintaining traditional protocols (TP), can limit gains and cause stagnation (Stone et al., 2007).

Introduction of CS could produce an adaptive stimulus allowing further gains in strength, RFD etc. During peaking phases aimed at improving power, CS training could enhance P output. Thus, CS training could be quite valuable for several aspects of the training process and possibly promote more consistent and perhaps superior gains when used appropriately.

Traditional loading (TL) schemes are believed to enhance adaptation, at least partly through acute fatigue. Acute fatigue could enhance motor unit recruitment (Joy et al., 2013), increase muscle (and whole body) metabolism and metabolite production (Folland et al., 2002; Girman et al., 2014; Gorostiaga et al., 2010a; Gorostiaga et al., 2010b; Iglesia-Soler et al., 2012; Iglesia-Soler et al., 2016; Oliver et al., 2016; Oliver et al., 2015; Stone et al., 2007) both of which may enhance adaptation to training. However, fatigue and increased production of metabolites as a primary stimulus for increased strength and power have both been questioned (Drinkwater et al., 2007; Folland et al., 2002). Folland et al. (2002) found that higher levels of training induced fatigue ( $4 \times 10$ to failure) did not provide additional benefits compared to a low fatigue protocol (40x1) with 30s of inter-repetition rest designed to minimize metabolic accumulation. Additionally, a tendency towards greater high-velocity gains in the low fatigue protocol was noticed, suggesting that velocity and perhaps power would be higher with greater inter-repetition rest. Indeed, further study on CS has demonstrated increased, or maintained force, RFD, velocity and power for CS compared to TP (Denton \& Cronin, 2006; Haff et al., 2003; Hansen et al., 2011a; Hansen et al., 2011b; Hardee et al., 2012; Iglesias-Soler et al., 2012; Joy et al., 2013; Lawton et al., 2006; Moir et al., 2013; Morales-Artacho et al., 2017; Oliver et al., 2016; Oliver et al., 2015; Tuffano et al., 2016a; Tuffano et al., 2016b).

Although CS protocols have been previously investigated, there are few studies describing both kinetic and kinematic characteristics and there are a number of limitations in these studies. A number of intra-set rest periods and exercises have been used (Denton \& Cronin, 2006; Drinkwater et al., 2007; 
Folland et al., 2002; Girman et al., 2014; Haff et al., 2003; Haff et al., 2016; Hansen et al., 2011a; Hansen et al., 2011b; Hardee et al., 2012a; Hardee et al., 2012b; Iglesias-Soler et al., 2012; Iglesias-Soler et al., 2016; Joy et al., 2013; Lawton et al., 2006; Matuszak et al., 2003; Mayo et al., 2014; Moir et al., 2013; Morales-Artacho et al., 2017; Moreno et al., 2014; Oliver et al., 2016; Oliver et al., 2015; Tuffano et al., 2016a; Tuffano et al., 2016b). Many of the existing studies investigated CS using machines, which could alter normal technique and may not be indicative of a typical athletic setting in which CS would be logically used. Studies (Drinkwater et al., 2007; Gorostiaga et al., 2010; Gorostiaga et al., 2012; MoralesArtacho et al., 2017; Moreno et al., 2014; Oliver et al., 2015) used untrained subjects which also may limit generalizability to trained populations. Additionally, most studies used only one type of instrumentation or used solely kinetic or kinematic data to study CS which may have created errors in calculation of variables, especially power. For example, Cormie et al. (2007a) indicate that using only kinetic data (e.g. force plate) may result in underestimating power while relying solely on kinematic data (e.g. potentiometers) can result in overestimation. Combining both kinetic and kinematic data appears to be superior when investigating force and related variables such as RFD, velocity and power (Cormie et al., 2007a).

The back squat is a commonly performed exercise, particularly in athletic settings. To the authors' knowledge, only two previous studies have used a combination of kinetic and kinematic data to study the squat in previously trained subjects using CS (Tuffano et al., 2016a; Tuffano et al., 2016b). The results indicate that CS can enhance maintenance of force related variables compared to TP.

The purpose of this study was to compare CS and TP training schemes in well-trained subjects. Both kinetic and kinematic collected data will be used to investigate the effects of CS as a programming tactic. 


\section{Comprehensive Review of the Literature}

Several key principles of resistance training include overload, specificity and variation. As athletes become highly trained, these principles often need manipulation to continue to drive adaptation (Stone et al., 2007). By utilizing more powerful stimuli, there is a larger disruption in homeostasis which requires more rest and variation to recover from training. One possible means of providing greater rest and variation in training may come in the form of cluster loading. Clusters, also known as inter-repetition rest, or rest-pause training, introduce rest during the set to minimize the effects of fatigue. It is commonly known that fatigue builds and performance decreases as a set continues into later repetitions (Gorostiaga et al., 2014). Additionally, it has been demonstrated that fatigue is not a necessary stimulus for additional gains in resistance training (Drinkwater et al., 2007; Folland et al., 2002). For example, Folland and colleagues (2002) compared a high fatigue protocol to a low fatigue protocol which aimed to minimize metabolic accumulation via inter-repetition rest. The results of this study indicate that higher training induced fatigue does not provide additional benefits when compared to the lower fatigue protocol. Additionally, there was a tendency towards greater highvelocity gains in the low fatigue protocol suggesting that power outputs may have been greater with inter-repetition rest. Drinkwater and colleagues (2007) demonstrated similar findings when investigating the effects of forced repetitions after failure on strength gains. They concluded that neither additional forced repetitions nor additional set volume improved the magnitude of strength gains once failure was reached. Therefore, fatigue may not be beneficial to training adaptations. It was also noted in a study by Sánchez and González-Badillo (2011) that velocity loss may be an objective means to quantifying neuro-muscular fatigue during resistance training. As noted earlier, power outputs had greater trends towards high-velocity gains with inter-repetition rest suggesting that cluster loading may help control fatigue during resistance training. 
As stated previously, $\mathrm{CL}$ has been widely investigated in a number of formats. These include different set and repetition schemes, loading schemes (intensity), rest intervals between repetitions and sets, exercises used and variables analyzed. The existing literature claims many benefits of using $\mathrm{CL}$ including but not limited to: increased power output, greater velocity maintenance, greater total work accomplished and subsequently greater time under tension and lower ratings of perceived exertion (RPE) (Denton \& Cronin, 2006; Haff et al., 2003; Hansen et al., 2011a; Hansen et al., 2011b; Hardee et al., 2012a; Hardee et al., 2012b; Iglesias-Soler et al., 2012; Joy et al., 2013; Lawton et al., 2006; Moir et al., 2013; Morales-Artacho et al., 2017; Moreno et al., 2014; Oliver et al., 2016; Oliver et al., 2015; Tuffano et al., 2016a; Tuffano et al., 2016b). There are many different studies which have shown greater power outputs and velocity while using cluster sets (Haff et al., 2003; Hansen et al., 2011a; Hansen et al., 2011b; Hardee et al., 2012a; Iglesias-Soler et al., 2012; Joy et al., 2013; Lawton et al., 2006; Mayo et al., 2014; Morales-Artacho et al., 2017; Moreno et al., 2014; Oliver et al., 2016; Oliver et al., 2015; Tuffano et al., 2016a; Tuffano et al., 2016b). One such study was done by Haff and colleagues (2003). In this study, clean pulls at $90 \%$ and $120 \%$ of 1 RM were analyzed for one set of five repetitions. Their findings suggest that cluster set configurations significantly higher peak velocity $(p=0.016)$ when compared to traditional loading. An interesting study carried out by Joy and Colleagues in 2013 demonstrated that not only did cluster sets result in greater mean power outputs $(p<0.05)$ during the later repetitions in each set when compared to traditional loading, but traditional loading also caused greater vastus lateralis electromyography EMG values $(p<0.05)$ during the later repetitions of a set (Joy et al., 2013). This may lend further support to the claim that cluster sets help to minimize the effects of fatigue while traditional sets may have to recruit more muscle fibers as fatigue sets in.

Several studies have attempted to investigate directly the metabolic implications of utilizing cluster loading tactics (Drinkwater et al., 2007; Escamilla et al., 2001; Hardee et al., 2012b). Iglesias-Soler et al. (2012) studied the acute effects of cluster loading on metabolic and performance measures. This 
study compared the effects of three sets to failure using traditional loading to three sets not to failure using cluster loading in the smith machine squat. The findings of this study show lower post-session blood lactate values $(p=0.001)$ in the cluster condition along with greater mean propulsive velocity ( $p=0.009)$ (Iglesias-Soler et al., 2012). In addition to this study, Girman et al (2014) compared the acute effects of cluster sets on metabolic and performance measures. Their findings further supported the findings of Iglesias et al. showing lower lactate values $(p<0.05)$ and greater maintenance of countermovement and standing long jump performance $(p<0.05)$. In a non-direct method of measuring the effects of cluster loading on fatigue, Hardee et al. compared the effects of clusters on ratings of perceived exertion (RPE). This protocol included three sets of six power cleans at 80\% 1RM utilizing either traditional loading, clusters with 20 s of inter-repetition rest or clusters with 40 s of inter-repetition rest. The results showed lower levels of decline in power for the cluster conditions indicating less fatigue. The cluster condition with 40s of rest resulted in significantly lower RPE compared to the other conditions which supports the claim that CL may help minimize the effects of fatigue (Hardee et al., 2012a).

In a series of studies, Oliver and colleagues investigated the effects of cluster loading in the back squat (Oliver et al., 2016; Oliver et al., 2015). These studies found that the cluster condition was able to complete a greater volume load than the TL scheme at the same absolute load. This was due to fatigue in the TL condition causing subjects to reduce the load in the later sets while CL conditions were able to maintain their load until completion. This suggests that CL may allow athletes to complete a training session at a higher absolute load for a given volume compared to traditional loading and could have large implications for strength development.

With this background in mind as to the effects of $\mathrm{CL}$, authors have turned their attention to the best means of utilizing this resistance training programming tactic. First, the intensity to be used must be considered. If the goal is to generate greater power output, a range of intensities may be considered. 
It is commonly known that power is the product of force and velocity, and thus has a spectrum ranging from higher force components to higher velocity components. Cormie et al. (2007c) investigated the intensity which maximized power output in the back squat, power clean and jump squat. The results indicated that all three exercises reached their maximal power output at different intensities and it should be considered whether the sport emphasizes higher velocity or force demands when determining the intensity to be used.

There are also many different forms of set and repetition schemes that can be used with cluster loading. Cluster sets of various repetition numbers have been previously investigated. For example, Lawton et al (2006) investigated three different cluster set configurations including single repetitions (6x1) with 20s inter-repetition rest, doubles (3x2) with 50 s of rest between the three sets, and triples (2x3) with 100 s of rest between the two sets. All configurations trained with a 6RM load. When compared to traditional loading, all cluster conditions showed higher total power outputs with no between group differences ( $p=0.96$ ) (Lawton et al., 2006). In another study, Tuffano et al. (2016a) compared traditional sets of twelve $(3 \times 12)$ to cluster sets of four $(3 \times 3 \times 4)$ and cluster sets of two $(3 \times 6 \times 2)$. The findings of this study indicate that both cluster configurations produced greater peak velocity, mean velocity, peak power and mean power when compared to traditional loading $(p<0.01)$. Additionally, it was found that cluster sets of two produced greater results for power and velocity than cluster sets of four $(p=0.02)$.

A multitude of exercises have been investigated utilizing $C L$. These exercises include: Back Squat (Girman et al., 2014; Hansen et al., 2011a; Iglesias-Soler et al., 2012; Iglesias-Soler et al., 2016; Matuszak et al., 2003; Mayo et al., 2014; Oliver et al., 2016; Oliver et al., 2015; Tuffano et al., 2016a; Tuffano et al., 2016b), Power Clean (Cormie et al., 2007a; Cormie et al., 2007b; Cormie et al., 2007c; Hansen et al., 2011b; Hardee et al., 2012a; Hardee et al., 2012b), Bench Press (Denton \& Cronin, 2006; Drinkwater et al., 2007; Lawton et al., 2006; Mayo et al., 2014), Deadlift (Moir et al., 2013), Clean Pulls (Girman et al., 
2014; Haff et al., 2003), leg extension (Folland et al., 2002), Vertical Jumps (Cormie et al., 2007a; Cormie et al., 2007b; Cormie et al., 2007c; Hansen et al., 2011a; Hansen et al., 2011b; Morales-Artacho et al., 2017; Moreno et al., 2014) and Bench Throw (Drinkwater et al., 2007). It is important to consider the desired outcomes of training when determining which exercise to select for training.

Lastly, several studies have provided possible explanations for the effects of CL. As mentioned earlier, fatigue builds as a set continues and as a session continues. It has been reported that lactate values are higher for traditional loading than $\mathrm{CL}$ suggesting a reliance on anaerobic glycolysis for energy (Denton \& Cronin, 2006; Girman et al., 2014; Oliver et al., 2015). Gorostiaga et al. (2012) has provided evidence of phosphocreatine depletion $(\mathrm{PCr}$ ) during resistance training sets of ten. $\mathrm{PCr}$ depletion was found to be significantly correlated with muscle lactate $\left(R^{2}=0.46\right)$ and inosine monophosphate $\left(R^{2}=\right.$ 0.44) (Gorostiaga et al., 2012). In a separate study, Gorostiaga et al. (2010) found that power declined during a second set of an exercise which coincided with a decreased adenosine tri-phosphate (ATP) utilization from the phosphagen energy system and an increase in ATP utilization from the anaerobic glycolysis energy system. It is commonly known that the phosphagen system is the most efficient energy system for intense activities such as resistance training and that PCr acts to support this system. Any decrease in PCr or phosphagen utilization would cause a decrease in energy efficiency and possibly decreased power output.

It has been suggested that cluster sets allow partial regeneration of $\mathrm{PCr}$ to better maintain power output (Gorostiaga et al., 2010; Gorostiaga et al., 2012; Haff et al., 2003). This claim was supported by Matuszak et als. (2003) finding that rest intervals as short as one minute were able to replenish adequate energy stores to repeat $1 \mathrm{RMs}$. This seems to suggest that very short rest intervals are able to replenish energy stores for very intense resistance training. With these findings in mind, it is possible that inter-set rest may allow partial replenishment of $\mathrm{PCr}$ which is a more efficient energy source and may allow for higher power outputs. 
There are several gaps in the existing literature surrounding cluster sets as a programming tactic. To our knowledge, there is only one semi-long-term training study using cluster loading. This study by Morales et al (2017) was only three weeks in duration and compared the effects of cluster and traditional loading on force-velocity profiles of counter movement jumps. The results of this study found no significant differences between the two conditions $(P=0.207, E S=0.31)$ but suggested that training with cluster loading is more effective at inducing velocity and power adaptations (Morales et al., 2017). Additionally, many of the studies cited in this review utilized machine-based exercises which is not indicative of typical strength and conditioning settings where cluster loading would most likely be used (Denton \& Cronin, 2006; Drinkwater et al., 2007; Folland et al., 2002; Ganzález-Badillo et al., 2014; Gorostiaga et al., 2010; Gorostiaga et al., 2012; Iglesias-Soler et al., 2012; Iglesias-Soler et al., 2016; Mayo et al., 2014; Morales et al., 2017; Oliver et al., 2016; Oliver et al., 2015; Sánchez \& GonzálezBadillo, 2011). Lastly, the means of estimating power outputs must be carefully considered when interpreting the results of many existing studies. Cormie et al (2007a) assert that only utilizing kinematic data leads to over-estimation of power outputs while relying on kinetic data only may lead to underestimation. The authors claim that both kinetic and kinematic data should be integrated to accurately estimate power. Of the included studies in this review, only eight studies integrated both kinetic and kinematic data measurement to estimate power (Gorostiaga et al., 2010; Gorostiaga et al., 2012; Hardee et al., 2012a; Hardee et al., 2012b; Moir et al., 2013; Oliver et al., 2016; Oliver et al., 2015; Tuffano et al., 2016a; Tuffano et al., 2016b). 


\section{CHAPTER 2}

\section{CLUSTER SET LOADING IN THE BACK SQUAT: KINETIC AND KINEMATIC IMPLICATIONS}

Alexander Wetmore ${ }^{1}$, John P. Wagle ${ }^{1}$, Matt L. Sams ${ }^{2}$, Christopher B. Taber ${ }^{3}$, Brad H. DeWeese ${ }^{1}$, Kimitake Sato ${ }^{1}$, and Michael H. Stone ${ }^{1}$

${ }^{1}$ Center of Excellence for Sport Science and Coach Education, Department of Sport, Exercise, Recreation, and Kinesiology, East Tennessee State University, Johnson City, TN, USA, ${ }^{2}$ Department of Exercise Science and Health Education, LaGrange College, LaGrange, GA 30240, ${ }^{3}$ Department of Physical Therapy and Human Movement Science, Sacred Heart University, Fairfield, CT, USA

(Formatted for submission to the Journal of Strength and Conditioning Research)

CORRESPONDING AUTHOR:

Alexander B. Wetmore

East Tennessee State University

Department of Sport, Exercise, Recreation, and Kinesiology

1081 John Robert Bell Dr.

Johnson City, TN, 37601

awetmore425@gmail.com 
TITLE: CLUSTER SET LOADING IN THE BACK SQUAT: KINETIC AND KINEMATIC IMPLICATIONS. 


\section{Abstract}

As athletes become well-trained, they require greater stimuli and variation to force adaptation. One means of adding additional variation is the use of cluster loading. Cluster loading involves introducing inter-repetition rest during a set which in theory may allow athletes to train at higher absolute intensities for the same volume. The purpose of this study was to investigate the kinetic and kinematic implications of cluster loading as a resistance training programming tactic compared to traditional loading. Eleven resistance trained males (age $=26.75 \pm 3.98$ yrs., Height $=181.36 \pm 5.96 \mathrm{~cm}$, Body Mass $=89.83 \pm 10.66 \mathrm{~kg}$, Relative Squat Strength=1.84 \pm 0.34 ) were recruited for this study. Each subject completed two testing sessions consisting of three sets of five back squats at $80 \%$ of their one repetition maximum with three minutes of inter-set rest. Cluster loading included 30s of inter-repetition rest with three minutes of inter-set rest. All testing was performed on dual-force plates sampling at $1000 \mathrm{~Hz}$ and the barbell was connected to four linear position transducers sampling at $1000 \mathrm{~Hz}$. Paired Samples ttests were used to determine differences between conditions with Cohen's $d$ effect sizes describing the magnitudes of any differences. Both conditions had similar values for peak force, concentric and eccentric average force $(p=.25, E S=0.09, p=0.25, E S=0.09, p=0.60, E S=0.04$ respectively). Cluster loading had significantly higher peak power $(p<0.001$, ES $=0.77)$, peak and average velocities $(p<0.001$, $E S=0.77, p<0.001, E S=0.81$ respectively $)$, shorter times to peak power and velocity $((p<0.001, E S=-0.68$, $p<0.001, E S=-0.68$ respectively) as well as greater maintenance of time to peak power $(p<0.001$, $E S=1.57)$. These results suggest cluster loading may be superior to traditional loading when maintaining power output and timepoint variables is the desired outcome of training.

Key Words: Training, Rest, Strength and Conditioning, Performance 


\section{INTRODUCTION}

Over the past several years, new advanced training (AT) methods have been proposed. These AT include accentuated eccentric loading, contrast sets, complex sets and cluster sets. Cluster sets (CS) use short rest periods between repetitions as well as typical rest periods between sets $(14,15)$. According to the SAID (specific adaptation to imposed demands) principle, changing variables within the application of an exercise elicits a specific response and subsequent adaptation given adequate recovery is provided (36). Thus, intra-set rest theoretically could allow CS to induce different adaptations to training by allowing for heavier loading at the same training volume (strength), potentiate explosiveness and power adaptations by maintenance of forces, rate of development (RFD), velocity, or power at a given load $(5,14,16,17,19,21,23,24,29,31,32,33,38,39)$. Thus, CS training could be useful for a variety of purposes such as enhancing the training effect by offering a greater stimulus or varying the stimulus to promote further adaptation. For example, training over a few years with little variation, such as can occur with maintaining traditional protocols (TP), can limit gains and cause stagnation (36). Introduction of CS could produce an adaptive stimulus allowing further gains in strength, RFD etc. During peaking phases aimed at improving power, CS training could enhance P output. Thus, CS training could be quite valuable for several aspects of the training process and possibly promote more consistent and perhaps superior gains when used appropriately.

Traditional loading (TL) schemes are believed to enhance adaptation, at least partly through acute fatigue. Acute fatigue could enhance motor unit recruitment (23), increase muscle (and whole body) metabolism and metabolite production $(9,10,12,13,21,22,32,33,36)$ both of which may enhance adaptation to training (36). However, fatigue and increased production of metabolites as a primary stimulus for increased strength and power have both been questioned $(7,9)$. Folland et al. (9) found that higher levels of training induced fatigue ( $4 \times 10$ to failure) did not provide additional benefits compared to a low fatigue protocol (40x1) with 30 s of inter-repetition rest designed to minimize metabolic 
accumulation. Additionally (Folland et al.) noted a tendency towards greater high-velocity gains in the low fatigue protocol; suggesting that velocity and perhaps power would be higher with greater interrepetition rest. Indeed, further study on CS has demonstrated increased, or maintained force, RFD, velocity and power for CS compared to TP $(5,14,16,17,19,21,23,24,29,30,32,33,38,39)$.

Although CS protocols have been previously investigated, there are few studies describing both kinetic and kinematic characteristics and there are a number of limitations in these studies. A number of intra-set rest periods and exercises have been used $(5,7,9,10,14,15,16,17,18,19,21,22,23,24,25,26,29,30,31,32,33,38,39)$. Many of the existing studies investigated CS using machines, which could alter normal technique and may not be indicative of a typical athletic setting in which CS would be logically used. Studies $(7,12,13,30,31,33)$ used untrained subjects which also may limit generalizability to trained populations. Additionally, most studies used only one type of instrumentation or used solely kinetic or kinematic data to study CS which may have created errors in calculation of variables, especially power. For example, Cormie et al. (1) indicate that using only kinetic data (e.g. force plate) may result in underestimating power while relying solely on kinematic data (e.g. potentiometers) can result in overestimation. Combining both kinetic and kinematic data appears to be superior when investigating force and related variables such as RFD, velocity and power (1).

The back squat is a commonly performed exercise, particularly in athletic settings. To the authors' knowledge, only one previous study has used a combination of kinetic and kinematic data to study the squat in previously trained subjects using CS $(38,39)$. The results indicate that CS can enhance maintenance of force related variables compared to TP. 
The purpose of this study was to compare CS and TP training schemes in well-trained subjects. Both kinetic and kinematic collected data will be used to investigate the effects of CS as a programming tactic.

\section{METHODS}

\section{Experimental Approach to the Problem}

The barbell back squat was chosen for this study because it a widely used exercise in strength and conditioning and has similar biomechanical and neuromuscular characteristics to a variety of sporting activities $(6,27,36)$. All subjects completed one pre-testing session and two experimental testing sessions. During the pre-testing session, subjects were tested on their one-repetition maximum in the back squat to establish experimental loads. Three days separated one-repetition maximum testing and experimental conditions. The cluster and traditional loading experimental conditions were randomly assigned with seven days separating the first and second testing session. Each experimental testing session was completed at the same time of day. A within-subject design was used to test the effect of rest-distribution on kinetic and kinematic performance variables.

\section{Subjects}

Eleven male subjects (age $=26.1 \pm 4.1$ years, height $=183.5 \pm 4.3 \mathrm{~cm}$, body mass $=92.5 \pm 10.5 \mathrm{~kg}$, back squat to body mass ratio $=1.8 \pm 0.3$ ) were recruited for this study. All subjects were required to have at least one year of resistance training experience with the back squat, be able to squat at least $1.3 x$ their body weight and have no major injuries within the previous three months. After explaining the risks and benefits of the study, all subjects signed informed consent documents before participation in accordance with the Institutional Review Board of the university. 


\section{Procedures}

\section{Body Composition}

Body composition was estimated by a certified ISAK anthropometrist using skinfolds and Harpenden skinfold calipers (Baty International, West Sussex, United Kingdom). Skinfold sites were: subscapular, triceps, chest, midaxillary, abdomen, iliac crest, and quadricep.

\section{Maximum Strength Testing}

Prior to each maximal strength testing session, each subject completed a standardized warm-up.

Subjects reported self-estimated one-repetition maximums on which warm-up repetitions were set. Warm-up repetitions began at $30 \%$ of their estimated maximum and ranged to $90 \%$ (Table 1). Subjects then performed their 1RM using a protocol modified from McBride et al. (2002) and Suchomel et al. (2015) $(26,35)$. The first recorded trial was at $90 \%$ of their reported 1 RM and jumps were made by $2.5-$ $5 \%$ until a maximum was reached. Full depth was defined as the subjects hip crease being below the patella and was verified by multiple certified strength and conditioning specialists.

\begin{tabular}{|l|l|}
\hline \multicolumn{2}{|l|}{ Table 2.1. Back squat warm-up } \\
\hline Sets $x$ repetitions $\mathrm{x}$ intensity (\% 1RM) & Rest interval \\
\hline $1 \times 5 \times 30 \%$ & $1 \mathrm{~min}$ \\
$1 \times 3 \times 50 \%$ & $1 \mathrm{~min}$ \\
$1 \times 2 \times 70 \%$ & $2 \mathrm{~min}$ \\
$1 \times 1 \times 80 \%$ & $3 \mathrm{~min}$ \\
$1 \times 1 \times 90 \%$ & $3 \mathrm{~min}$ \\
$1 \mathrm{RM}$ attempts & $3 \mathrm{~min}$ \\
\hline
\end{tabular}

\section{Experimental Conditions}

All subjects completed an identical standardized dynamic warm-up to the 1RM testing. Subjects were randomly assigned to either the traditional set or cluster set condition at least 2 days following the 1RM testing. The opposite testing condition was separated by one week. Subjects completed $3 \times 5$ sets at $80 \%$ of the established $1 \mathrm{RM}$ with three minutes rest between sets in the traditional loading (TL) condition. For the cluster condition (CL), 30s of inter-repetition rest was given with three minutes inter-set rest. 
Subjects were instructed to stand up as explosively as possible from the bottom of the squat. Depth was confirmed by multiple certified strength and conditioning specialists.

\section{$\underline{\text { Instrumentation }}$}

Data was collected using dual force plates $(2 \times 91 \mathrm{~cm} \times 45.5116 \mathrm{~cm}$ force plates, RoughDeck HP, Rice Lake, WI) sampling at $1000 \mathrm{~Hz}$ while connected to four linear position transducers (PT101-0100-H141120, Celesco Measurement Specialties, Chatsworth, CA) to collect kinematic data. All data was simultaneously integrated into LabVIEW (version 7.1, National Instruments.

\section{Data Analysis}

All data was analyzed using a custom-designed application (R Studio vers. 3.4.1.) Kinetic variables analyzed included: peak power, peak force, average power, average force, and total work. Kinematic variables included: peak velocity, average velocity, time to peak power (TTPP), and time to peak velocity (TTPV). Paired sample t-tests were used to determine effects of condition on the above listed variables. Cohen's d effect sizes were calculated for each dependent variable to determine the magnitude and meaningfulness of the differences between dependent variables across load conditions. For practical significance, effect sizes were interpreted with magnitude thresholds of $0-0.2,0.2-0.6,0.6-1.2,1.2-2.0$, and 2.0 and above as trivial, small, moderate, large, and very large (20). Percent changes were calculated as the change in value from repetition one to repetition five of each set and were averaged across all subjects. Statistical analysis was performed using a statistic software package (JASP vers. 0.8.2.0. Significance was defined as a $p$-value of $p \leq 0.05$.

\section{RESULTS}

Kinetic Variables

There were no significant differences across all sets in peak force $(p=0.25, E S=0.09)$ or average Force $(p=0.25, E S=0.09)$ between conditions. Cluster loading conditions did have statistically higher 
total work across all sets than traditional loading conditions $(p<0.001, E S=0.28)$. Additionally, Cluster loading had a very large effect on Peak power $(p<0.001, E S=0.77)$. Kinetic results are shown in Table 2. Traditional loading had statistically larger peak power losses across all sets compared to cluster loading $(p=0.005, E S=0.52)$ with average losses of $8.5 \%, 9.3 \%$ and $8.3 \%$ compared to $3.3 \%,+3.0 \%$ and $4.1 \%$ across sets 1, 2, and 3, respectively. Peak Power changes across all three sets are shown seen in Figure 1.

Figure 2.1 Peak power changes across three sets

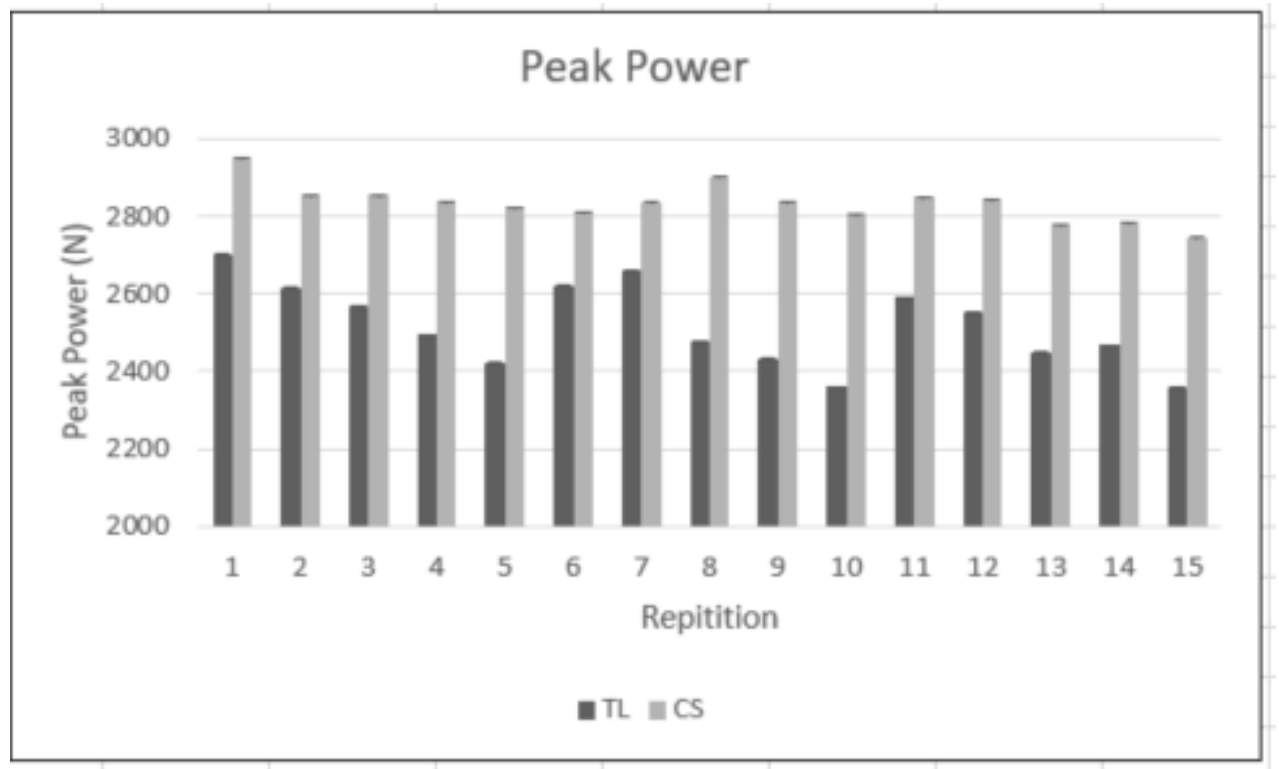

Table 2.2 Full-Rep kinetic variables

\begin{tabular}{|l|l|l|l|l|}
\hline & $\begin{array}{l}\text { Traditional } \\
\text { Loading Mean }\end{array}$ & Mean & P-Value & $\begin{array}{l}\text { Effect Size } \\
\text { (Cohen's d) }\end{array}$ \\
\hline Peak Force (N) & 3002 & 3012 & 0.249 & 0.091 \\
\hline Peak Power (W) & 2518 & 2834 & $<0.001$ & 0.770 \\
\hline Total Work & 3035.674 & 3068.183 & $<0.001$ & 0.279 \\
\hline
\end{tabular}

Kinematic Variables

Clusters displayed statistically higher peak velocities ( $p<0.001, E S=0.77)$, and average velocities $(p<0.001, E S=0.81)$. Additionally, clusters had statistically lower times to peak power $(p<0.001, E S=-0.68)$ 
and peak velocity $(p<0.001, E S=-0.68)$ compared to traditional loading. Complete kinematic results are listed in Table 3. Traditional loading showed statistically greater increases in time to peak power $(p<0.001, E S=1.57)$ across all sets when compared to cluster loading with average increases of $31.6 \%$, $37.5 \%$ and $38.4 \%$ for sets 1,2 , and 3 , respectively whereas cluster loading conditions displayed only $6.5 \%, 9.3 \%$, and $11.6 \%$ increases for sets 1,2 , and 3 , respectively. Traditional loading conditions also demonstrated larger, although non-statistically significant, increases in time to peak velocity $(p=.329$, $E S=0.17$ ) with average increases of $30.3 \%, 35.9 \%$ and $36.5 \%$ for sets 1,2 , and 3 , respectively whereas cluster loading showed only $6.2 \%, 9.2 \%$ and $11.4 \% \%$ increases for sets 1,2 , and 3 , respectively. Changes in time to peak power and velocity are shown in Figures 2 and 2 respectively.

Table 2.3 Kinematic Variables

\begin{tabular}{|c|c|c|c|c|}
\hline & $\begin{array}{l}\text { Traditional } \\
\text { Loading Mean }\end{array}$ & $\begin{array}{l}\text { Cluster Loading } \\
\text { Mean }\end{array}$ & P-Value & $\begin{array}{l}\text { Effect Size } \\
\text { (Cohen's d) }\end{array}$ \\
\hline $\begin{array}{l}\text { Con. Peak Velocity } \\
(\mathrm{m} / \mathrm{s})\end{array}$ & 1.013 & 1.106 & $<0.001$ & 0.767 \\
\hline $\begin{array}{l}\text { Con. Average } \\
\text { Velocity (m/s) }\end{array}$ & 0.489 & 0.541 & $<0.001$ & 0.805 \\
\hline $\begin{array}{l}\text { Con. Peak } \\
\text { Acceleration } \\
\left(\mathrm{m} / \mathrm{s}^{2}\right)\end{array}$ & 4.292 & 4.421 & 0.030 & 0.172 \\
\hline $\begin{array}{l}\text { Con. Average } \\
\text { Acceleration } \\
\left(\mathrm{m} / \mathrm{s}^{2}\right)\end{array}$ & -0.006 & -0.007 & 0.002 & 0.241 \\
\hline
\end{tabular}




\begin{tabular}{|l|l|l|l|l|}
\hline Time to Peak & 1.267 & 1.134 & $<0.001$ & 0.682 \\
Power (s) & & & & \\
\hline Time to Peak & 1.311 & 1.178 & $<0.001$ & 0.684 \\
Velocity (s) & & & & \\
\hline
\end{tabular}

Figure 2.2 Changes in time to peak power across three sets

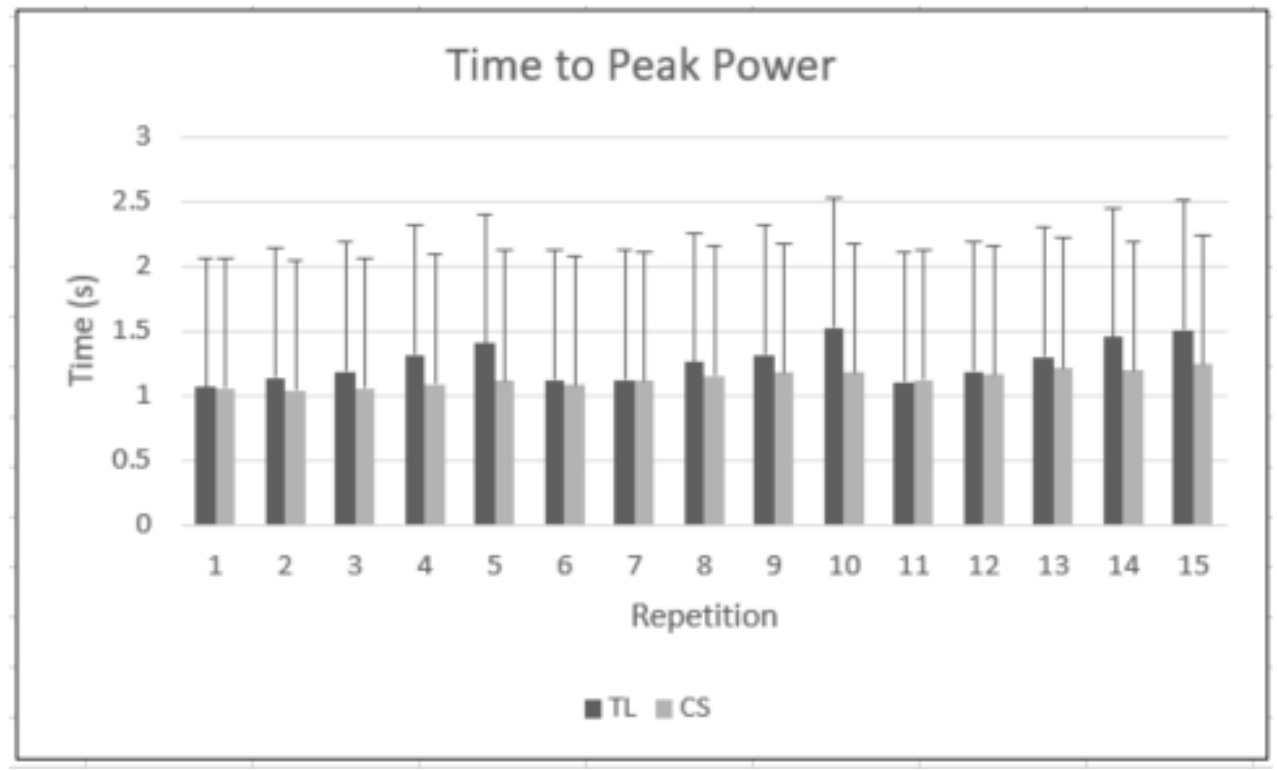

Figure 2.3 Changes in time to peak velocity across three sets

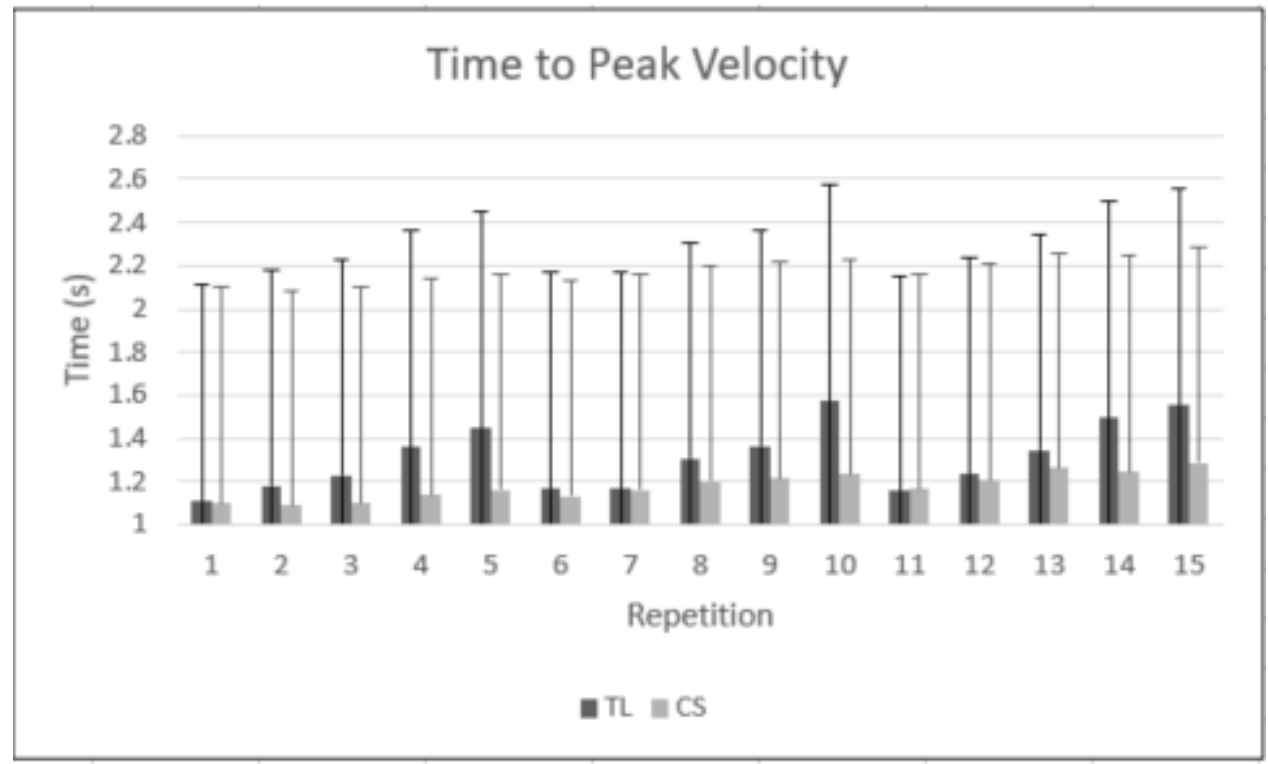




\section{Discussion}

This study is only the second study to the authors' knowledge that has investigated both kinetic and kinematic variables during cluster loading of the barbell back squat $(36,37)$. Cormie et al. suggest using only kinetic data may underestimate power output while relying on kinematic data may result in overestimation in the back squat, power lean and jump squat (1). Therefore, we used a combination of both kinetic and kinematic data to best estimate power outputs. Additionally, many previous studies have used machines for testing, however this may cause alterations in exercise technique and may not accurately reflect how most athletic populations train $(5,21,22,26,32,33,34)$.

The results of our study support the hypothesis that cluster set loading would produce higher peak and average power outputs, and velocities when compared to traditional loading. Tuffano et al. found similar results with cluster sets showing greater peak and mean velocity, peak and mean power with compared to traditional $(38,39)$. Because of the effect on power, cluster sets may prove to be a valuable tool to enhance power, particularly during the later stages of a sequential training plan that emphasizes power production $(6,36)$. Evidence has consistently shown that stronger athletes are more powerful then weaker athletes $(36,37)$. The inclusion of cluster sets once the focus of training has shifted towards power development warrants consideration.

This leads us to consider what the mechanism is that allows regeneration of power with interset rest. It has been suggested that cluster sets allow partial or complete regeneration of Phosphocreatine $(\mathrm{PCr})$ to better maintain power output $(12,13,14)$. This is supported by Matuszak et als. finding that very short rest intervals as low as one minute are sufficient to repeat 1RM attempts (25). It is commonly known that high intensity exercise relies on ATP as its main energy source. However, these energy stores are limited and may be depleted during resistance training. $\mathrm{PCr}$ helps to sustain this energy system but is also limited and may be depleted. Therefore, it is possible that inter-set rest may allow partial replenishment of PCr which is a more efficient energy source and may allow for higher 
power outputs. It has also been reported that lactate values are higher for TL than CS suggesting a reliance on anaerobic glycolysis for energy $(5,10,33)$. Gorostiaga also reported higher reliance on lactate during the last five repetitions in a set of ten (12). These data support the claim that CS would allow less metabolic disturbance than TL.

Cluster set loading may provide beneficial training adaptations, especially for athletic populations. The results of this study and others suggest that cluster set loading consistently demonstrates greater peak and average power outputs when compared to traditional loading. Although these findings are acute, chronic adaptations to cluster loading have been previously investigated (30). Morales et al. showed three weeks of cluster training in the counter-movement jump caused greater adaptations in velocity and power (30). Additionally, athletes must be sure to maximize movement intent when trying to stimulate beneficial training adaptations (11). Percent changes are calculated as the change in a variable from the start to the end of each set. Gonzales and colleagues showed greater gains in $1 \mathrm{RM}$, and average velocity in the bench press when training with maximal intent (11). In a later study, Gonzales and colleagues also showed training with maximal intent may have caused beneficial changes in myosin heavy chain isoforms, excitability, firing rate, neural drive etc., all of which support the development of power (11). Lastly, because many sports are time-limited (e.g. ground contact times in sprinting etc.), time to peak power (TTPP) and time to peak velocity (TTPV) are important to consider. Because of their greater maintenance of both TTPP and TTPV they allow athletes to train in a more explosive manner for the entirety of the set. As mentioned earlier, this may also lend support for the inclusion of traditional sets earlier in a training year and cluster sets later in a sequential phase of the training year. Traditional sets cause athletes to spend more time accelerating the bar, as seen in their longer TTPP and TTPV. This would seem to support the goals of strength endurance and general strength development. As you approach the later stages of a periodized model, shorter TTPP and TTPV are desired as the emphasis of training has shifted towards speed-strength development. 
One limitation of the current study is that we investigated only one repetition scheme and one inter-repetition rest interval. Many possible configurations of cluster sets can be used. Others have previously investigated cluster sets of different configurations but utilized different rest-intervals $(5,7,10,14,15,16,17,18,19,20,25,31)$. Future investigation should be performed to determine the optimal set and rest interval configurations to maximize training adaptations.

\section{Practical Application}

This study provides insight into a means of manipulating training variables to achieve the desired adaptations to training. In keeping with the principle of specificity, coaches wanting to maximize power should use programming tactics which emphasize power output. Cluster sets may provide a means of developing strength while maximizing power output by using greater absolute loads for the same volume as TL. This study demonstrates that cluster set loading maximizes power output via greater velocity both within and across sets. Therefore, cluster sets may provide a means of directing training towards greater power development. Coaches may consider utilizing cluster sets during training phases in which power is the desired training goal. 


\section{$\underline{\text { References }}$}

1. Cormie, P., J.M. McBride, and G.O. McCaulley, Validation of power measurement techniques in dynamic lower body resistance exercises. J Appl Biomech, 2007. 23(2): p. 103-18.

2. Cormie, P., G.O. McCaulley, and J.M. McBride, Power versus strength-power jump squat training: influence on the load-power relationship. Med Sci Sports Exerc, 2007. 39(6): p. 996-1003.

3. Cormie, P., et al., Optimal loading for maximal power output during lower-body resistance exercises. Med Sci Sports Exerc, 2007. 39(2): p. 340-9.

4. Cunanan, A.J., et al., The General Adaptation Syndrome: A Foundation for the Concept of Periodization. Sports Med, 2018: p. 1-11.

5. Denton, J. and J. Cronin, The kinematic, kinetic and blood lactate profiles of continuous and intra-set rest loading schemes. Journal of Strength and Conditioning Research, 2006. 20(3): p. 528-534.

6. DeWeese, B.H., et al., The training process: Planning for strength-power training in track and field. Part 2: Practical and applied aspects. Journal of Sport and Health Science, 2015. 1(7).

7. Drinkwater, E., et al., Increased Number of Forced Repetitions Does Not Enhance Strength Development With Resistance Training. The Journal of Strength and Conditioning Research, 2007. 21(3): p. 841-847.

8. Escamilla, R.F., et al., Effects of technique variations on knee biomechanics during the squat and leg press. Med Sci Sports Exerc, 2001. 33(9): p. 1552-66.

9. Folland, J.P., et al., Fatigue is not a necessary stimulus for strength gains during resistance training. British Journal of Sports Medicine, 2002. 36: p. 370-374.

10. Girman, J.C., et al., Acute effects of a cluster-set protocol on hormonal, metabolic and performance measures in resistance trained males. European Journal of Sport Science, 2014. 14(2).

11. González-Badillo, J.J., et al., Maximal intended velocity training induces greater gains in bench press performance than deliberately slower half-velocity training. Eur J Sport Sci, 2014. 14(8): p. $772-$ 81.

12. Gorostiaga, E.M., et al., Anaerobic energy expenditure and mechanical efficiency during exhaustive leg press exercise. PLoS One, 2010. 5(10): p. e13486.

13. Gorostiaga, E., M., et al., Energy Metabolism during Repeated Sets of Leg Press Exercise Leading to Failure or Not. PLoS ONE, 2012. 7(7).

14. Haff, G.G., et al., Effects of different set configurations on barbell velocity and displacement during a clean pull. J Strength Cond Res, 2003. 17(1): p. 95-103. 
15. Haff, G.G. Cluster Sets - Current Methods for Introducing Variation into Training Sets. in National Strength and Conditioning Association National Conference. 2016. New Orleans, LA.

16. Hansen, K.T., J.B. Cronin, and M.J. Newton, The effect of cluster loading on force, velocity, and power during ballistic jump squat training. Int J Sports Physiol Perform, 2011. 6(4): p. 455-68.

17. Hansen, K.T., et al., Does cluster loading enhance lower body power development in preseason preparation of elite rugby union players? J Strength Cond Res, 2011. 25(8): p. 2118-26.

18. Hardee, J.P., et al., Effect of inter-repetition rest on ratings of perceived exertion during multiple sets of the power clean. Eur J Appl Physiol, 2012. 112(8): p. 3141-7.

19. Hardee, J.P., et al., Effect of interrepetition rest on power output in the power clean. J Strength Cond Res, 2012. 26(4): p. 883-9.

20. Hopkins, W.G., et al., Progressive statistics for studies in sports medicine and exercise science. Med Sci Sports Exerc, 2009. 41(1): p. 3-13.

21. Iglesias-Soler, E., et al., Acute effects of distribution of rest between repetitions. Int J Sports Med, 2012. 33(5): p. 351-8.

22. Iglesias-Soler, E., et al., Inter-repetition rest training and traditional set configuration produce similar strength gains without cortical adaptations. J Sports Sci, 2016. 34(15): p. 1473-84.

23. Joy, J.M., et al., Power Output and Electromyography Activity of the Back Squat Exercise with Cluster Sets. Journal of Sport Science

2013. 1: p. 37-45

24. Lawton, T.W., J.B. Cronin, and R.P. Lindsell, Effect of interrepetition rest intervals on weight training repetition power output. J Strength Cond Res, 2006. 20(1): p. 172-6.

25. Matuszak, M.E., et al., Effect of rest interval length on repeated 1 repetition maximum back squats. J Strength Cond Res, 2003. 17(4): p. 634-7.

26. Mayo, X., E. Iglesias-Soler, and M. Fernández-Del-Olmo, Effects of set configuration of resistance exercise on perceived exertion. Percept Mot Skills, 2014. 119(3): p. 825-37.

27. McBride, J.M., et al., Relationship between maximal squat strength and five, ten, and forty yard sprint times. J Strength Cond Res, 2009. 23(6): p. 1633-6.

28. McBride, J.M., et al., The effect of heavy-vs. light-load jump squats on the development of strength, power, and speed. J Strength Cond Res, 2002. 16(1): p. 75-82.

29. Moir, G.L., et al., Effect of cluster set configurations on mechanical variables during the deadlift exercise. J Hum Kinet, 2013. 39: p. 15-23. 
30. Morales-Artacho, A.J., et al., Influence of a Cluster Set Configuration on the Adaptations to ShortTerm Power Training. The Journal of Strength and Conditioning Research, 2017.

31. Moreno, S.D., et al., Effect of cluster sets on plyometric jump power. J Strength Cond Res, 2014. 28(9): p. 2424-8.

32. Oliver, J.M., et al., Velocity Drives Greater Power Observed During Back Squat Using Cluster Sets. J Strength Cond Res, 2016. 30(1): p. 235-43.

33. Oliver, J.M., et al., Acute response to cluster sets in trained and untrained men. Eur J Appl Physiol, 2015. 115(11): p. 2383-93.

34. Sánchez-Medina, L. and J.J. González-Badillo, Velocity loss as an indicator of neuromuscular fatigue during resistance training. Med Sci Sports Exerc, 2011. 43(9): p. 1725-34.

35. Schoenfeld, B.J., Squatting kinematics and kinetics and their application to exercise performance. J Strength Cond Res, 2010. 24(12): p. 3497-506.

36. Stone, M.H., M. Stone, and W.A. Sands, Principles and Practice of Resistance Training. 2007, Champaign, IL: Human Kinetics.

37. Suchomel, T.J., P. Comfort, and M.H. Stone, Weightlifting pulling derivatives: rationale for implementation and application. Sports Med, 2015. 45(6): p. 823-39.

38. Tufano, J.J., et al., Maintenance of Velocity and Power With Cluster Sets During High-Volume Back Squats. Int J Sports Physiol Perform, 2016. 11(7): p. 885-892.

39. Tufano, J.J., et al., Maintenance of Velocity and Power With Cluster Sets During High-Volume Back Squats. Int J Sports Physiol Perform, 2016. 11(7): p. 885-892. 


\section{CHAPTER 3}

\section{SUMMARY AND FUTURE DIRECTIONS}

The purpose of this study was to investigate the effects of both cluster and traditional loading schemes on kinetic and kinematic outputs in the back squat. To the authors knowledge, this is only the second study that has investigated both kinetic and kinematic effects of CS in the back squat. The results of this study indicate that CS had statistically greater total work $(p<0.001, E S=0.28)$, peak power $(p<1.001, E S=0.77)$, peak velocity $(p<0.001, E S=0.77)$, average velocity $(p<0.001, E S=0.81)$. CS also had lower times to peak power $(p<0.001, E S=0.68)$ and times to peak velocity $(p<0.001, E S=0.68)$. Additionally, TL had statistically greater increases in times to peak power across all sets $(p<0.001$, $E S=1.57)$ indicating the onset of fatigue across a set. The results of this study expand upon the findings of previous studies by providing a complete description of the kinetic and kinematic effects of CS and may help the application of this programming tactic. These results suggest that CS provides a greater acute stimulus when strength and power adaptation is the desired outcome of training.

Power is widely considered the most important factor relating to sporting success (Stone et al., 2007). Because of their greater power and velocity outputs, CS may be a useful programming tactic during a training phase which is aimed at enhancing these qualities. Additionally, greater maintenance of timepoint variables in CS may further enhance the acute stimulus of resistance training.

In the current study, only one set and repetition scheme were investigated. Future studies dealing with the effects of CS should investigate other set and repetition schemes to determine the optimal configuration. Additionally, this study only investigated one rest interval. Many possible rest intervals are commonly used in strength and conditioning settings and should be further studied to determine the interval which maximizes the beneficial effects of CS. Lastly, there was no direct investigation into the mechanism behind the enhanced outputs of CS. It has previously been suggested that partial regeneration of $\mathrm{PCr}$ may contribute to these effects, but this claim warrants further study. 


\section{$\underline{\text { References }}$}

Cormie, P., McBride, J. M., \& McCaulley, G. O. (2007). Validation of power measurement techniques in dynamic lower body resistance exercises. J Appl Biomech, 23(2), 103-118.

Cormie, P., McCaulley, G. O., \& McBride, J. M. (2007). Power versus strength-power jump squat training: influence on the load-power relationship. Med Sci Sports Exerc, 39(6), 996-1003. doi:10.1097/mss.0b013e3180408e0c

Cormie, P., McCaulley, G. O., Triplett, N. T., \& McBride, J. M. (2007). Optimal loading for maximal power output during lower-body resistance exercises. Med Sci Sports Exerc, 39(2), 340-349. doi:10.1249/01.mss.0000246993.71599.bf

Cunanan, A. J., DeWeese, B. H., Wagle, J. P., Carroll, K. M., Sausaman, R., Hornsby, W. G. I., . . Stone, M. H. (2018). The General Adaptation Syndrome: A Foundation for the Concept of Periodization. Sports Med, 1-11.

Denton, J., \& Cronin, J. (2006). The kinematic, kinetic and blood lactate profiles of continuous and intraset rest loading schemes. Journal of Strength and Conditioning Research, 20(3), 528-534.

DeWeese, B. H., Hornsby, G., Stone, M., \& Stone, M. H. (2015). The training process: Planning for strength-power training in track and field. Part 2: Practical and applied aspects. Journal of Sport and Health Science, 1(7).

Drinkwater, E., Lawton, T. W., McKenna, M. J., \& Pyne, D. B. (2007). Increased Number of Forced Repetitions Does Not Enhance Strength Development With Resistance Training. The Journal of Strength and Conditioning Research, 21(3), 841-847.

Escamilla, R. F., Fleisig, G. S., Zheng, N., Lander, J. E., Barrentine, S. W., Andrews, J. R., . . Moorman, C. T. (2001). Effects of technique variations on knee biomechanics during the squat and leg press. Med Sci Sports Exerc, 33(9), 1552-1566.

Folland, J. P., Irish, C. S., Roberts, J. C., Tarr, J. E., \& Jones, D. A. (2002). Fatigue is not a necessary stimulus for strength gains during resistance training. British Journal of Sports Medicine, 36, 370-374.

Girman, J. C., Jones, M. T., Matthews, T. D., \& Wood, R. J. (2014). Acute effects of a cluster-set protocol on hormonal, metabolic and performance measures in resistance trained males. European Journal of Sport Science, 14(2).

González-Badillo, J. J., Rodríguez-Rosell, D., Sánchez-Medina, L., Gorostiaga, E. M., \& Pareja-Blanco, F. (2014). Maximal intended velocity training induces greater gains in bench press performance than deliberately slower half-velocity training. Eur J Sport Sci, 14(8), 772-781.

doi:10.1080/17461391.2014.905987 
Gorostiaga, E., M., Navarro-Ameqzueta, I., A.L. Calbet, J., Hellsten, Y., Cusso, R., Guerrero, M., . . . Izquierdo, M. (2012). Energy Metabolism during Repeated Sets of Leg Press Exercise Leading to Failure or Not. PLOS ONE, 7(7).

Gorostiaga, E. M., Navarro-Amézqueta, I., Cusso, R., Hellsten, Y., Calbet, J. A., Guerrero, M., . . . Izquierdo, M. (2010). Anaerobic energy expenditure and mechanical efficiency during exhaustive leg press exercise. PLoS One, 5(10), e13486. doi:10.1371/journal.pone.0013486

Haff, G. G. (2016). Cluster Sets - Current Methods for Introducing Variation into Training Sets. Paper presented at the National Strength and Conditioning Association National Conference, New Orleans, LA.

Haff, G. G., Whitley, A., McCoy, L. B., O'Bryant, H. S., Kilgore, J. L., Haff, E. E., . . Stone, M. H. (2003). Effects of different set configurations on barbell velocity and displacement during a clean pull. $J$ Strength Cond Res, 17(1), 95-103.

Hansen, K. T., Cronin, J. B., \& Newton, M. J. (2011). The effect of cluster loading on force, velocity, and power during ballistic jump squat training. Int J Sports Physiol Perform, 6(4), 455-468.

Hansen, K. T., Cronin, J. B., Pickering, S. L., \& Newton, M. J. (2011). Does cluster loading enhance lower body power development in preseason preparation of elite rugby union players? J Strength Cond Res, 25(8), 2118-2126. doi:10.1519/JSC.0b013e318220b6a3

Hardee, J. P., Lawrence, M. M., Utter, A. C., Triplett, N. T., Zwetsloot, K. A., \& McBride, J. M. (2012). Effect of inter-repetition rest on ratings of perceived exertion during multiple sets of the power clean. Eur J Appl Physiol, 112(8), 3141-3147. doi:10.1007/s00421-011-2300-x

Hardee, J. P., Triplett, N. T., Utter, A. C., Zwetsloot, K. A., \& Mcbride, J. M. (2012). Effect of interrepetition rest on power output in the power clean. J Strength Cond Res, 26(4), 883-889. doi:10.1519/JSC.0b013e3182474370

Hopkins, W. G., Marshall, S. W., Batterham, A. M., \& Hanin, J. (2009). Progressive statistics for studies in sports medicine and exercise science. Med Sci Sports Exerc, 41(1), 3-13. doi:10.1249/MSS.0b013e31818cb278

Iglesias-Soler, E., Carballeira, E., Sánchez-Otero, T., Mayo, X., Jiménez, A., \& Chapman, M. L. (2012). Acute effects of distribution of rest between repetitions. Int J Sports Med, 33(5), 351-358. doi:10.1055/s-0031-1299699

Iglesias-Soler, E., Mayo, X., Río-Rodríguez, D., Carballeira, E., Fariñas, J., \& Fernández-Del-Olmo, M. (2016). Inter-repetition rest training and traditional set configuration produce similar strength gains without cortical adaptations. J Sports Sci, 34(15), 1473-1484.

doi:10.1080/02640414.2015.1119299

Joy, J. M., Oliver, J. M., McCleary, S. A., Lowery, R. P., \& Wilson, J. M. (2013). Power Output and Electromyography Activity of the Back Squat Exercise with Cluster Sets. Journal of Sport Science $1,37-45$ 
Lawton, T. W., Cronin, J. B., \& Lindsell, R. P. (2006). Effect of interrepetition rest intervals on weight training repetition power output. J Strength Cond Res, 20(1), 172-176. doi:10.1519/R-13893.1

Matuszak, M. E., Fry, A. C., Weiss, L. W., Ireland, T. R., \& McKnight, M. M. (2003). Effect of rest interval length on repeated 1 repetition maximum back squats. J Strength Cond Res, 17(4), 634-637.

Mayo, X., Iglesias-Soler, E., \& Fernández-Del-Olmo, M. (2014). Effects of set configuration of resistance exercise on perceived exertion. Percept Mot Skills, 119(3), 825-837.

doi:10.2466/25.29.PMS.119c30z3

McBride, J. M., Blow, D., Kirby, T. J., Haines, T. L., Dayne, A. M., \& Triplett, N. T. (2009). Relationship between maximal squat strength and five, ten, and forty yard sprint times. J Strength Cond Res, 23(6), 1633-1636. doi:10.1519/JSC.0b013e3181b2b8aa

McBride, J. M., Triplett-McBride, T., Davie, A., \& Newton, R. U. (2002). The effect of heavy- vs. light-load jump squats on the development of strength, power, and speed. J Strength Cond Res, 16(1), 7582.

Moir, G. L., Graham, B. W., Davis, S. E., Guers, J. J., \& Witmer, C. A. (2013). Effect of cluster set configurations on mechanical variables during the deadlift exercise. J Hum Kinet, 39, 15-23. doi:10.2478/hukin-2013-0064

Morales-Artacho, A. J., Padial, P., Garcia-Ramos, A., Perez-Castilla, A., \& Feriche, B. (2017). Influence of a Cluster Set Configuration on the Adaptations to Short-Term Power Training. The Journal of Strength and Conditioning Research.

Moreno, S. D., Brown, L. E., Coburn, J. W., \& Judelson, D. A. (2014). Effect of cluster sets on plyometric jump power. J Strength Cond Res, 28(9), 2424-2428. doi:10.1519/JSC.0000000000000585

Oliver, J. M., Kreutzer, A., Jenke, S., Phillips, M. D., Mitchell, J. B., \& Jones, M. T. (2015). Acute response to cluster sets in trained and untrained men. Eur J Appl Physiol, 115(11), 2383-2393. doi:10.1007/s00421-015-3216-7

Oliver, J. M., Kreutzer, A., Jenke, S. C., Phillips, M. D., Mitchell, J. B., \& Jones, M. T. (2016). Velocity Drives Greater Power Observed During Back Squat Using Cluster Sets. J Strength Cond Res, 30(1), 235-243. doi:10.1519/JSC.0000000000001023

Schoenfeld, B. J. (2010). Squatting kinematics and kinetics and their application to exercise performance. J Strength Cond Res, 24(12), 3497-3506. doi:10.1519/JSC.0b013e3181bac2d7

Stone, M. H., Stone, M., \& Sands, W. A. (2007). Principles and Practice of Resistance Training. Champaign, IL: Human Kinetics.

Suchomel, T. J., Comfort, P., \& Stone, M. H. (2015). Weightlifting pulling derivatives: rationale for implementation and application. Sports Med, 45(6), 823-839. doi:10.1007/s40279-015-0314-y 
Sánchez-Medina, L., \& González-Badillo, J. J. (2011). Velocity loss as an indicator of neuromuscular fatigue during resistance training. Med Sci Sports Exerc, 43(9), 1725-1734.

doi:10.1249/MSS.0b013e318213f880

Tufano, J. J., Conlon, J. A., Nimphius, S., Brown, L. E., Banyard, H. G., Williamson, B. D., ... Haff, G. G. (2017). Cluster Sets: Permitting Greater Mechanical Stress Without Decreasing Relative Velocity. Int J Sports Physiol Perform, 12(4), 463-469. doi:10.1123/ijspp.2015-0738

Tufano, J. J., Conlon, J. A., Nimphius, S., Brown, L. E., Seitz, L. B., Williamson, B. D., \& Haff, G. G. (2016). Maintenance of Velocity and Power With Cluster Sets During High-Volume Back Squats. Int J Sports Physiol Perform, 11(7), 885-892. doi:10.1123/ijspp.2015-0602 
VITA

ALEXANDER B. WETMORE

Education:

Professional Experience:

Professional Certifications:

Abstracts:
M.S. Sport Science and Coach Education

East Tennessee State University, Johnson City, TN, 2018

B.S. Exercise Science

Marian University, Indianapolis, IN, 2016

Zionsville High School, Zionsville, IN, 2012

Graduate Research Assistant

East Tennessee State University, Johnson City, TN, 2016-2018

Graduate Strength \& Conditioning Coach

East Tennessee State University, Johnson City, TN, 2016-2018

Sport Performance Trainer, Acceleration Indiana West

Indianapolis, IN, 2015-2016

NSCA Certified Strength and Conditioning Specialist, 2016

ISAK Level 1 Certified Anthropometrist, 2017

USATF Level 1 Certified Coach, 2017

Alex Wetmore, Joe Walters, Jason P. McCleery, Taylor Portman (2016). Sport science on a budget: a cost-effective approach for athlete monitoring. ETSU Coaches \& Sport Science College, December 2016

John P. Wagle, Kevin M. Carroll, Aaron J. Cunanan, Alexander Wetmore, Garett E. Bingham, Robert W. Sausaman, Brad H. DeWeese, Kimitake Sato, Christopher B. Taber, Charles A. Stuart, and Michael H. Stone (2017). Comparison of The Relationship Between Lying and Standing Ultrasonography Measures of Pennation Angle with Isometric Peak and Rate of Force Production. ETSU Coaches \& Sport Science College, December 2017.

Alex Wetmore, Kelsey Scarborough, Justin Taylor, Jason P. McCleery (2017). Relationship Between Training Monotony and Neuromuscular Performance in National Collegiate Athletics Association Soccer Players. ETSU Coaches \& Sport Science College, December 2017. 
Peer Reviewed Publications: Wagle, J.P., et al., Comparison of the Relationship between Lying and Standing Ultrasonography Measures of Muscle Morphology with Isometric and Dynamic Force Production Capabilities. Sports, 2017. $5(88)$.

Honors and Awards:

First place poster, sport science category, ETSU Coaches and Sport Science College, December 2017.

Second place poster, coach education category, ETSU Coaches and Sport Science College, December 2017.

Marian University Summa Cum Laude, 2016

Marian University Dean's List 2012-2016

Professional Conferences Attended:

NSCA Coaches Conference, January 2017

ETSU Coaches and Sport Science College, December 2017

ETSU Coaches and Sport Science College, December 2016

NSCA Indiana State Clinic, July 2016

Courses Taught:

PEXS 4061 Exercise Fitness Testing, ETSU, 2017

PHED 1140 Strength and Conditioning, ETSU, 2017 